\title{
Quantum phase diffusions of a spinor condensate
}

\author{
S. Yi, Ö. E. Müstecaplıŏlu, and L. You \\ School of Physics, Georgia Institute of Technology, Atlanta, GA 30332-0430, USA
}

(Dated: October 29, 2018)

\begin{abstract}
We discuss the quantum phases and their diffusion in a spinor- 1 atomic Bose-Einstein condensate. For ferromagnetic interactions, we obtain the exact ground state distribution of the phase fluctuations corresponding to the total atom number $(N)$, the magnetization $(\mathcal{M})$, and the alignment (or hypercharge) $(Y)$ of the system. The mean field ground state is shown to be stable against these fluctuations, which dynamically recover the two continuous symmetries associated with the conservation of $N$ and $\mathcal{M}$ as in current experiments.
\end{abstract}

Since the observation of Bose-Einstein condensation of trapped atomic clouds [1, 2], the coherence properties of the condensate has become the focus of many theoretical studies [3, 4, 5, 6, 7, 8, 9]. Within the mean-field theory, it is commonly assumed that the condensate can be described by a $U(1)$ symmetry breaking field [10, 11], equivalent to a coherent state assumption of the ground state. (see Refs. 10, 11] for discussions of $U(1)$-symmetric approaches). Although quite successful in providing theoretical understanding to many experimental observations, such a coherent state assumption is not necessarily consistent with real experimental situations, where the fluctuations of the atom numbers are difficult to control 12, 13]. A coherent state leads to a Poisson distribution of atoms. For a ground state with average of $N$ atoms, the associated number fluctuations are of the order $\sqrt{N}$. As was initially pointed out in Refs. 3, 4], this number fluctuation of a coherent state condensate leads to the "diffusion" (or spreading) of its initial phase. In a scalar condensate, this diffusion, a dynamic attempt to restore the $U(1)$ symmetry of the interacting atomic system, can be studied in terms of a zero mode, or the Goldstone mode of the condensate [3]. More physically meaningful discussions in terms of the relative phase of two condensates were studied soon afterwards [5, 6, 67, 8]. Experimentally, starting with the remarkable direct observation of the first order coherence in an interference experiment 14, direct correlations between number and phase fluctuations were observed with a condensate in a periodic potential [15], and more recently, in the remarkable Mott insulating state [16] obtained by loading a superfluid condensate into an optical lattice 17].

The emergence of spinor-1 condensates [18, 19] (of atoms with hyperfine quantum number $F=1$ ) has created new opportunities to understand quantum coherence and the associated number/phase dynamics in a three component condensate [20, 21]. In this paper, we investigate the quantum phase dynamics of a spinor-1 condensate due to atom number fluctuations. We will focus on ferromagnetic interactions, when the condensate wave functions for the three spin components share the same spatial mode 22]; in this case, the phase fluctuations translate into fluctuations of the direction of the macroscopic condensate spin.

We consider a system of $N$ spin-1 bosonic atoms interacting via only s-wave scattering [23, 24, 25, 26]. A weak magnetic field $B$ (as always exists in an experiment) fixes the quantization z-axis such that quadratic Zeeman effect can be neglected. In the second-quantized form, the Hamiltonian is 23, 24.

$$
\begin{aligned}
H= & \sum_{i} \int d \vec{r} \psi_{i}^{\dagger}(\vec{r})\left[-\frac{\hbar^{2} \nabla^{2}}{2 M}+V_{\mathrm{ext}}(\vec{r})-\hbar \omega_{L} \mathbf{F}_{z}\right] \psi_{i}(\vec{r}) \\
& +\frac{c_{0}}{2} \sum_{i, j} \int d \vec{r} \psi_{i}^{\dagger}(\vec{r}) \psi_{j}^{\dagger}(\vec{r}) \psi_{i}(\vec{r}) \psi_{j}(\vec{r}) \\
& +\frac{c_{2}}{2} \sum_{i, j, k, l} \int d \vec{r} \psi_{i}^{\dagger}(\vec{r}) \psi_{j}^{\dagger}(\vec{r}) \overrightarrow{\mathbf{F}}_{i k} \cdot \overrightarrow{\mathbf{F}}_{j l} \psi_{l}(\vec{r}) \psi_{k}(\vec{r}), \quad(1)
\end{aligned}
$$

where $\psi_{j}(\vec{r})(j=+, 0,-)$ denotes the annihilation operator for the $j$-th component of a spinor- 1 field. The trapping potential $V_{\text {ext }}(\vec{r})$ is assumed harmonic and spinindependent. The Larmor precessing frequency is $\omega_{L}=$ $B \mu_{B} / \hbar$ with $\mu_{B}$ the magnetic dipole moment for state $\left|F=1, M_{F}=1\right\rangle$. The pseudo potential coefficients are $c_{0}=4 \pi \hbar^{2}\left(a_{0}+2 a_{2}\right) / 3 M$ and $c_{2}=4 \pi \hbar^{2}\left(a_{2}-a_{0}\right) / 3 M$, with $a_{0}\left(a_{2}\right)$ the s-wave scattering length for two spin-1 atoms in the combined symmetric channel of total spin 0 (2). $M$ is the mass of atom, and $\overrightarrow{\mathbf{F}}$ is the spin 1 operator [25]. Hamiltonian (1) is invariant under $\mathrm{U}(1)$ gauge transformation $e^{i \theta}$ and $\mathrm{SO}(3)$ spin rotations $\mathcal{U}(\alpha, \beta, \tau)=$ $e^{-i F_{z} \alpha} e^{-i F_{y} \beta} e^{-i F_{z} \tau}$ (for $B=0$ ) [23]. A non-zero $B$ or the conservation of magnetization $\mathcal{M}=N_{+}-N_{-}$reduces the $\mathrm{SO}(3)$ to its subgroup $\mathrm{SO}(2)$ generated by $e^{-i F_{z} \alpha}$.

Following the Bogoluibov theory we assume there exist three 'large' condensate components $\phi_{j}$ around which we study the small quantum fluctuations (off-condensate excitations) via

$$
\psi_{j}(\vec{r})=\sqrt{N} \phi_{j}(\vec{r})+\delta \psi_{j}(\vec{r}),
$$

where $N \int d \vec{r}\left|\phi_{j}\right|^{2}=N_{j}$ is the number of condensed atoms in component $j$. At near zero temperatures, higher than quadratic terms of $\delta \psi_{j}(\vec{r})$ in the Hamiltonian (1) are neglected. The assumed mean field ground state $\sqrt{N} \phi_{j}(\vec{r})$ breaks both continuous symmetries U(1) and $\mathrm{SO}(2)$, thus we expect to observe multiple zero energy Goldstone modes [3].

In the first order, we obtain the usual coupled GrossPitaevskii equation (GPE) for the condensate modes $\phi_{j}(\vec{r})$. The quantum fluctuations $\delta \psi_{j}$ obey the usual 
Bogoluibov-de Gennes equations (BdGEs) 27]. The condensate number fluctuations, can be studied through the number fluctuation operators 3 ]

$$
\begin{aligned}
P_{j} & \equiv \int d \vec{r}\left[\phi_{j}^{*}(\vec{r}) \delta \psi_{j}(\vec{r})+\phi_{j}(\vec{r}) \delta \psi_{j}^{\dagger}(\vec{r})\right] \\
& \approx \frac{1}{\sqrt{N}} \int d \vec{r}\left[\psi_{j}^{\dagger}(\vec{r}) \psi_{j}(\vec{r})-N \phi_{j}^{*}(\vec{r}) \phi_{j}(\vec{r})\right]
\end{aligned}
$$

Using the GPEs and the BdGEs [27] their equations of motion are [3] ,

$$
i \hbar \dot{P}_{ \pm}=-i \hbar \dot{P}_{0} / 2
$$

from which we find that both $P_{\text {tot }}=P_{+}+P_{0}+P_{-}$and $P_{+}-P_{-}$are constants of motion, an obvious outcome since the Hamiltonian (11) commutes with operators of both the total number of atoms and magnetization.

We define the phase operator

$$
Q_{j}=i \hbar \int d \vec{r}\left[\theta_{j}^{*}(\vec{r}) \delta \psi_{j}(\vec{r})-\theta_{j}(\vec{r}) \delta \psi_{j}^{\dagger}(\vec{r})\right],
$$

with $\theta_{j}(\vec{r})$ the associated phase mode functions. Denote $Q_{\text {tot }}=Q_{+}+Q_{0}+Q_{-}$, the canonical quantization condition $\left[Q_{\text {tot }}, P_{\text {tot }}\right]=i \hbar$ is satisfied if the constraint $J_{+}+J_{0}+J_{-} \equiv 1$ is enforced, where $J_{j} \equiv$ $\int d \vec{r}\left[\theta_{j}^{*}(\vec{r}) \phi_{j}(\vec{r})+\right.$ c.c. $]$. As for a scalar or binary condensate [6]

$$
\frac{d Q_{\mathrm{tot}}}{d t}=N \tilde{u} P_{\mathrm{tot}}
$$

We note that $\left[Q_{j}, P_{k}\right]=i \hbar \delta_{j k} J_{j},\left[\delta \psi_{j}(\vec{r}), P_{k}\right]=$ $\delta_{j k} \phi_{j}(\vec{r})$, and $\left[\delta \psi_{j}(\vec{r}), Q_{k}\right]=-i \hbar \delta_{j k} \theta_{j}(\vec{r})$. These lead to $\delta \psi_{j}(\vec{r}) \approx \theta_{j}(\vec{r}) P_{j} / J_{j}+\phi_{j}(\vec{r}) Q_{j} / i \hbar J_{j}[\underline{6}$, []], which helps to define the complete dynamic equations for the number and phase fluctuations [28].

Including both the initial and time phases, the ground state wave functions can be generally expressed as $\left|\phi_{j}(\vec{r})\right| e^{-i\left(\mu-j \hbar \omega_{L}\right) t / \hbar+i \alpha_{j}}$ with a common chemical potential $\mu$, a Lagrange multiplier enforcing the conservation of atom numbers. Minimization of the total energy Eq. (11) leads to $\alpha_{+}+\alpha_{-}-2 \alpha_{0}=0$ [29]. It was further proven in Ref. 22 that the steady state solution takes the same spatial mode for each of its three spin components (for ferromagnetic interactions), i.e.

$$
\phi_{j}(\vec{r})=\sqrt{n_{j}} \phi(\vec{r}) e^{i \alpha_{j}},
$$

where the real-valued mode function $\phi(\vec{r})$ is normalized to unity, and governed by an equivalent scalar GPE

$$
\left[-\frac{\hbar^{2} \nabla^{2}}{2 M}+V_{\mathrm{ext}}(\vec{r})+c_{+} N \phi^{2}(\vec{r})\right] \phi(\vec{r})=\mu \phi(\vec{r})
$$

of a scattering length $a_{2}$ (note $c_{+}=c_{0}+c_{2} \propto a_{2}$ ). Define $n_{j}=N_{j} / N$ and $m=\mathcal{M} / N$ as the relative atom numbers and relative magnetization, $n_{ \pm}=(1 \pm m)^{2} / 4$ and $n_{0}=$ $\left(1-m^{2}\right) / 2$ in the ferromagnetic ground state [22, 25].
Similarly, the phase functions share the same spatial mode and can be generally expressed as

$$
\theta_{j}(\vec{r}, t)=\sqrt{n_{j}} \theta(\vec{r}) e^{-i\left(\mu-j \hbar \omega_{L}\right) t / \hbar+i \alpha_{j}}
$$

with $\theta(\vec{r})$ governed by the following equation

$$
\left[-\frac{\hbar^{2} \nabla^{2}}{2 M}+V_{\text {ext }}-\mu+3 c_{+} N \phi^{2}\right] \theta(\vec{r})=N \tilde{u} \phi(\vec{r}) .
$$

The normalization $\int d \vec{r} \phi(\vec{r}) \theta(\vec{r})=1 / 2$ determines the Goldstone parameter $\tilde{u}$, which in the Thomas-Fermi (TF) limit is $\tilde{u}=\left[c_{+} /\left(4 \pi a_{\mathrm{ho}}^{3} / 3\right)\right]\left(a_{\mathrm{ho}} / 15 a_{2}\right)^{3 / 5} N^{-3 / 5}$, with $a_{\mathrm{ho}}$ the ground state size $\left(\hbar / M \omega_{\text {ho }}\right)^{1 / 2}$ of a harmonic trap with a frequency $\omega_{\text {ho }}=\left(\omega_{x} \omega_{y} \omega_{z}\right)^{1 / 3}$.

Equations (8) and (10) are identical to their counterparts for a scalar condensate [3]. In the ferromagnetic ground state, individual atomic spins align along the same direction, thus they collide only in the symmetric total spin $F=2$ channel. To conserve the magnetization with respect to the $B$-field direction, the condensate spin direction is simply tilted at an angle $\theta=\cos ^{-1} m$. If this direction is taken as the quantization axis, then the spinor condensate behaves essentially as a scalar one $[22,23,30]$ when the $B$-field is zero.

With the mode functions for atom number and phase fluctuations, we find the zero mode dynamics can be expressed in terms of an associated Hamiltonian. After laborious calculation we arrive at 28]

$$
\frac{H_{\text {zero }}}{N}=\mathbf{p}^{\prime T} \mathcal{A} \mathbf{p}^{\prime}+\mathbf{q}^{\prime T} \mathcal{B} \mathbf{q}^{\prime}
$$

with $\mathbf{p}^{\prime T}=\left(p_{+}^{\prime}, p_{0}^{\prime}, p_{-}^{\prime}\right), \mathbf{q}^{\prime T}=\left(q_{+}^{\prime}, q_{0}^{\prime}, q_{-}^{\prime}\right)$, redefined as canonically conjugated variables $p_{j}^{\prime} \equiv P_{j}$ and $q_{j}^{\prime} \equiv$ $Q_{j} / J_{j} \cdot \mathcal{A} \& \mathcal{B}$ are two Hermitian and positive-definite matrices involving only parameters of the system. Thus $H_{\text {zero }} \geq 0$, the assumed ground state, a mean field symmetry breaking state with coherent condensate amplitudes $\sqrt{N} \phi_{j}(\vec{r})$ is stable. The associated quantum fluctuations of atom numbers can be studied with the linearization approximation Eq. (2). Matrices $\mathcal{A} \& \mathcal{B}$ are simplified with $\mathcal{O}_{\phi \theta} \equiv \int d \vec{r} \phi^{2}(\vec{r}) \theta^{2}(\vec{r})$ and $\mathcal{O}_{\phi \phi} \equiv$ $\int d \vec{r} \phi^{4}(\vec{r})$. We also find $\tilde{u}=\varepsilon+4 c_{+} \mathcal{O}_{\phi \theta}$ with $N \varepsilon=$ $2 \int d \vec{r} \theta(\vec{r})\left[\mathcal{L}-\mu+c_{+} N \phi^{2}(\vec{r})\right] \theta(\vec{r})$ a non-negative quantity.

We note that matrix $\mathcal{B}$ is diagonalized by an orthogonal transformation $q_{N}=\left(q_{+}^{\prime}+q_{0}^{\prime}+q_{-}^{\prime}\right) / \sqrt{3}, q_{M}=$ $\left(q_{+}^{\prime}-q_{-}^{\prime}\right) / \sqrt{2}$, and $q_{Y}=\left(q_{+}^{\prime}-2 q_{0}^{\prime}+q_{-}^{\prime}\right) / \sqrt{6}$ 31]. The corresponding $p_{N}, p_{M}$, and $p_{Y}$ are respectively fluctuations of the total number of atoms, the magnetization, and the alignment. With these new collective operators, the zero mode Hamiltonian becomes

$$
\begin{aligned}
\frac{H_{\text {zero }}}{N}= & a p_{N}^{2}+b p_{M}^{2}+c p_{Y}^{2} \\
& +\alpha p_{N} p_{Y}+\beta p_{M} p_{Y}+\gamma p_{N} p_{M}+\eta q_{Y}^{2}
\end{aligned}
$$

where all the coefficients are listed in the Appendix. Replacing $p_{j}$ by $-i \hbar \partial / \partial q_{j}$, Hamiltonian (12) leads to the 

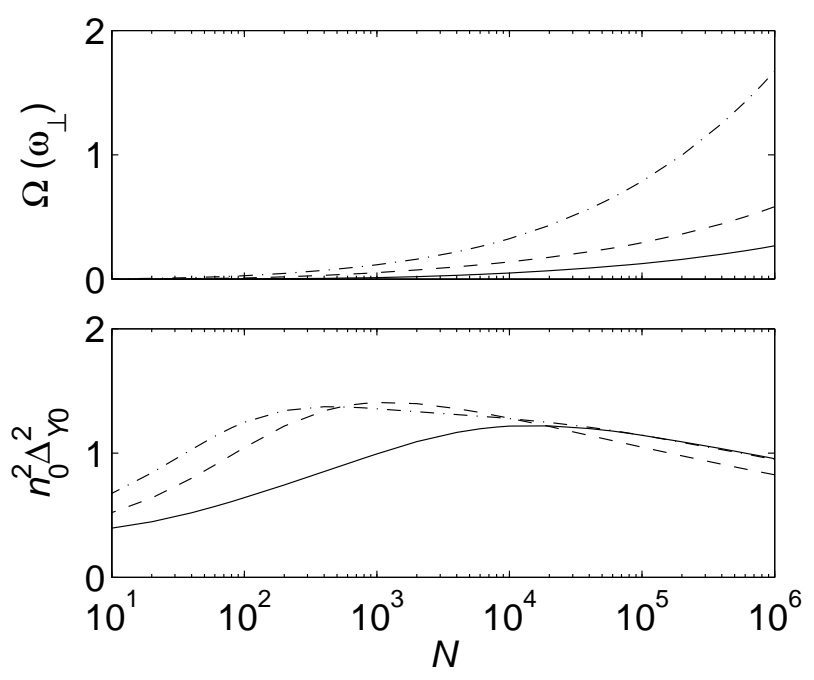

FIG. 1: The N-dependence of $\Omega$ and $3 n_{0}^{2} \Delta_{Y 0}^{2}$ for a ${ }^{87} \mathrm{Rb}$ condensate in a cylindrically symmetric trap with $\omega_{x}=\omega_{y}=$ $\omega_{\perp}=(2 \pi) 100(\mathrm{~Hz})$ and $\omega_{z}=\lambda \omega_{\perp} . \quad \lambda=0.1$ (solid line), $\lambda=1.0$ (dashed line), and $\lambda=10.0$ (dash-dotted line).

ground state distribution of fluctuations

$$
\varphi_{0}\left(q_{N}, q_{M}, q_{Y}\right)=\frac{1}{\sqrt{\sqrt{\pi} \hbar \Delta_{Y 0}}} \exp \left(-\frac{q_{Y}^{2}}{2 \hbar^{2} \Delta_{Y 0}^{2}}\right),
$$

with a width

$$
\Delta_{Y 0}^{2}=\frac{1}{3 n_{0}^{2}}\left(\frac{\varepsilon-4 c_{2} \mathcal{O}_{\phi \theta}}{-c_{2} \mathcal{O}_{\phi \phi}}\right)^{1 / 2},
$$

and the zero point energy $E_{0} \equiv \hbar \Omega / 2=N\left[-c_{2} \mathcal{O}_{\phi \phi}(\varepsilon-\right.$ $\left.\left.4 c_{2} \mathcal{O}_{\phi \theta}\right)\right]^{1 / 2}$. We find $n_{0}^{2} \Delta_{Y 0}^{2}=\sqrt{7 / 10} / 3$ and $\Omega \propto$ $-\left(c_{2} / \pi a_{\mathrm{ho}}^{3}\right)\left(a_{\mathrm{ho}} / a_{2}\right)^{3 / 5} N^{2 / 5}$ in the TF limit (and also taking the small $\varepsilon \rightarrow 0$ ). Figure 1 shows selected results for the $N$-dependence of $\Omega$ and $\Delta_{Y 0}^{2}$. While $\Omega$ increases monotonically with $N$, the ground state width $\Delta_{Y 0}^{2}$ for $q_{Y}$ peaks at some intermediate values of atom numbers and eventually saturates in the limit of large $N$ (or strong interactions).

The above ground state distribution of $H_{\text {zero }}$ can easily be understood. For a condensate with fixed total number of atoms and magnetization, the conservations of $N$ and $\mathcal{M}$ require $\left\langle p_{N}^{2}\right\rangle=\left\langle p_{M}^{2}\right\rangle=0$, which lead to $\left\langle q_{N}^{2}\right\rangle=\left\langle q_{M}^{2}\right\rangle=\infty$, i.e., completely diffused phases; The ferromagnetic interaction, nevertheless, prepares a correlated ground state such that both distributions for $p_{Y}$ and $q_{Y}$ take a Gaussian form with $\left\langle p_{Y}^{2}\right\rangle=1 / 2 \Delta_{Y 0}^{2}$ and $\left\langle q_{Y}^{2}\right\rangle=\hbar^{2} \Delta_{Y 0}^{2} / 2$. Such a distribution in $p_{Y}$ and $q_{Y}$ is in fact the minimal uncertainty coherent state consistent with the symmetry breaking ground state. However, it is experimentally difficult to produce such a condensate having fixed atom numbers and phase fluctuations. For any initial state, the dynamic solution $\mathbf{x}(t)=\mathcal{T}(t) \mathbf{x}(0)$ as governed by the Hamiltonian Eq.
(12) for $\mathbf{x}^{T}(t)=\left[p_{N}(t), p_{M}(t), p_{Y}(t), q_{N}(t), q_{M}(t), q_{Y}(t)\right]$ can be used. This solution has a simple structure [28]; in addition to oscillating terms of the forms $\cos \Omega t$ and $\sin \Omega t$, the phase fluctuations of $q_{N}$ and $q_{M}$ also contain diffusion terms proportional to $N t$, which indicates the linearization approximation of Eq. (2) is valid only for a finite duration.

Finally, let's consider the diffusion of the direction of the condensate spin $\vec{f}(\vec{r}, t) \equiv \sum_{i j} \psi_{i}^{\dagger}(\vec{r}, t) \overrightarrow{\mathbf{F}}_{i j} \psi_{j}(\vec{r}, t)$. Because all three spin components share the same spatial mode function 22], individual spins of Bose condensed atoms are parallel for ferromagnetic interactions, i.e. they act as a macroscopic magnetic dipole pointing along the same direction (independent of the spatial coordinates $): \vec{f}_{0}(\vec{r}, t) \propto\left(\sqrt{1-m^{2}} \cos \Theta,-\sqrt{1-m^{2}} \sin \Theta, m\right)$ while undergoing precessing with respect to the z-axis. $\Theta=\alpha_{+}-\alpha_{0}+\omega_{L} t$. As the phase dynamics attempts to restore the $\mathrm{U}(1)$ and $\mathrm{SO}(2)$ symmetries of the system, their respective initial values become irrelevant. The fluctuation becomes $\delta \vec{f}(t)=\int d \vec{r}\left[\vec{f}(\vec{r}, t)-\vec{f}_{0}(\vec{r}, t)\right]$ with a zero average. Using $\sigma_{N}^{2}, \sigma_{M}^{2}$, and $\sigma_{Y}^{2}$ to denote the initial variances of $N, \mathcal{M}$, and $Y$, we find in spherical coordinates $(\hat{r}, \hat{\theta}, \hat{\phi})\left(\right.$ note $\left.\sin \theta=\sqrt{1-m^{2}}\right), \delta f_{r}(t) \equiv \delta f_{r}(0)$ and $\delta f_{\theta}(t) \equiv \delta f_{\theta}(0)$. Both are fixed constants

$$
\begin{aligned}
\left\langle\left[\delta f_{r}(t)\right]^{2}\right\rangle & =3 \sigma_{N}^{2}, \\
\left\langle\left[\delta f_{\theta}(t)\right]^{2}\right\rangle & =\frac{1}{1-m^{2}}\left(3 m^{2} \sigma_{N}^{2}+2 \sigma_{M}^{2}\right),
\end{aligned}
$$

(due to conservations of $N$ and $\mathcal{M}$ ) when respective fluctuations in $N, \mathcal{M}$, and $Y$ are uncorrelated. In the azimuthal $\hat{\phi}$ direction a simple phase diffusion results

$$
\delta f_{\phi}(t)=\delta f_{\phi}(0)-R_{d} t
$$

with $\delta f_{\phi}(0)=\sqrt{1-m^{2}}\left[\sqrt{2} q_{M}(0)+\sqrt{6} m q_{Y}(0)\right] / 2 \hbar$, and a diffusion rate $R_{d}=2 \varepsilon N \delta f_{\theta}(0) / \hbar$, proportional to $\delta f_{\theta}(0)$, (which can be explained in terms of the single axis twisting of $T_{z}^{2}$ in the isospin subspace of a spior-1 condensate [31]). The $N$ - and $\lambda$-dependence of the diffusion parameter $N \varepsilon$ are shown in Fig. 2 for a ${ }^{87} \mathrm{Rb}$ condensate. In the TF limit and applying the results of Ref. 32], we find that $\varepsilon=\left(4 \hbar^{2} / 5 M R^{2}\right) \ln \left(R / 1.3 a_{\mathrm{ho}}\right) \propto N^{-2 / 5}$, generally a very small quantity. $R=a_{\mathrm{ho}}\left(15 a_{2} / a_{\mathrm{ho}}\right)^{1 / 5} N^{1 / 5}$ is the TF radius. Typically, the dephasing rate is a fraction of $\omega_{\perp}$ for a condensate of $\sim 10^{5}$ atoms and $\lambda \sim 1$, i.e. its macroscopic spin direction is lost in a few cycles of trap oscillation. Figure 2 also indicates that the faster a ferromagnetic condensate diffuses its pointing direction along the precession direction, the larger and more tightly confined a condensate is.

When $B=0$, zero mode dynamics becomes much simpler if the condensate spin direction is taken as z-axis. Although there still exist more than one Goldstone mode in this case, the mean field ground state corresponds to all atoms in state $|+\rangle$ [23, 30]. Thus to leading order, the only relevant fluctuation is that of the total number of atoms (corresponding to the U(1) symmetry), and 


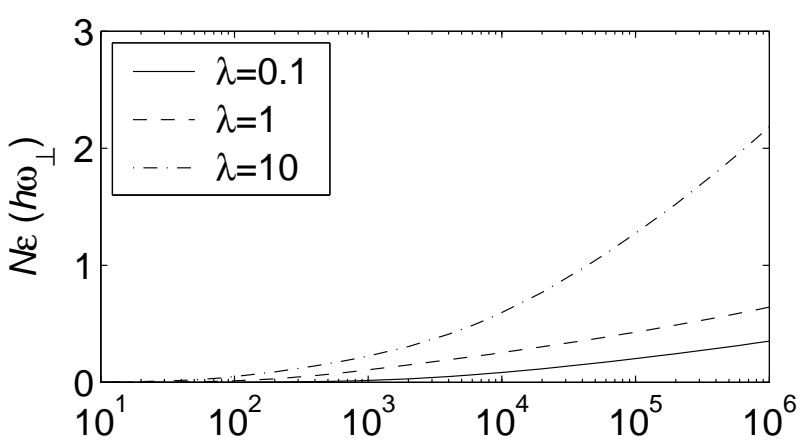

FIG. 2: The same as in Fig. 1 but for $N \varepsilon$.

the phase diffusion dynamics becomes the same as in a scalar condensate [3]. Our theory as developed in this paper does reduce to this simple limit.

In conclusion, we have studied in detail quantum phase diffusions of a spinor- 1 condensate with ferromagnetic interactions. The condensate ground state is very simple, all three spin components have the same spatial mode function and their associated phase functions are also identical. We have constructed the zero mode Hamiltonian for the condensate number and phase fluctuations, and solved for the ground state distribution of these fluctuations when both $N$ and $\mathcal{M}$ are conserved. Furthermore, we have obtained analytically the dynamic number and phase fluctuations relating to both the quantum phase diffusion and the initial distribution of these fluctuations. We have identified a quantum phase diffusion coefficient for the pointing direction of the condensate spin and recovered its small-time quadratic t-dependent spreading.

This work is supported by the NSF grant No. PHYS0140073 and by a grant from NSA, ARDA, and DARPA under ARO Contract No. DAAD19-01-1-0667.

\section{APPENDIX A: COEFFICIENTS IN EQ. (12)}

The coefficients in the zero mode Hamiltonian (12) are

$$
\begin{aligned}
a= & \frac{1}{4 n_{0}^{2}}\left[\frac{\varepsilon}{3}\left(5+3 m^{2}\right)+2 \mathcal{O}_{\phi \theta}\left[3 c_{0}\left(1-m^{2}\right)^{2}\right.\right. \\
& \left.\left.\quad+\frac{8}{3} c_{2}\left(1-3 m^{2}\right)\right]\right], \\
b= & \frac{1}{2 n_{0}^{2}}\left[\varepsilon\left(1+m^{2}\right)-8 c_{2} \mathcal{O}_{\phi \theta} m^{2}\right] \\
c= & \frac{1}{3 n_{0}^{2}}\left(\varepsilon-4 c_{2} \mathcal{O}_{\phi \theta}\right), \\
\alpha= & \frac{\sqrt{2}}{6 n_{0}^{2}}\left(1+3 m^{2}\right)\left(\varepsilon-4 c_{2} \mathcal{O}_{\phi \theta}\right), \\
\beta= & -\frac{2 m}{\sqrt{3} n_{0}^{2}}\left(\varepsilon-4 c_{2} \mathcal{O}_{\phi \theta}\right), \\
\gamma= & -\frac{2 m}{n_{0}^{2}} \sqrt{\frac{2}{3}}\left[\varepsilon-c_{2} \mathcal{O}_{\phi \theta}\left(1+3 m^{2}\right)\right] \\
\eta= & -3 c_{2} n_{0}^{2} \mathcal{O}_{\phi \phi} / \hbar^{2} .
\end{aligned}
$$

[1] M. H. Anderson et al., Science 269, 198 (1995).

[2] K. B. Davis et al., Phys. Rev. Lett. 75, 3969 (1995).

[3] M. Lewenstein and L. You, Phys. Rev. Lett. 77, 3489 (1996).

[4] E. M. Wright et al., Phys. Rev. Lett. 77, 2158 (1996).

[5] A. Imamoglu et al., Phys. Rev. Lett. 78, 2511 (1997).

[6] P. Villain et al., J. Mod. Opt. 44, 1775 (1997).

[7] C. K. Law et al., Phys. Rev. Lett. 79, 3105 (1997).

[8] P. Villain and M. Lewenstein, Phys. Rev. A 59, 2250 (1999).

[9] J. Javanainen and M. Wilkens, Phys. Rev. Lett. 78, 4675 (1997).

[10] C. W. Gardiner, Phys. Rev. A 56, 1414 (1997).

[11] Y. Castin and R. Dum, Phys. Rev. A 57, 3008 (1998).

[12] H. W. Xiong et al., Phys. Rev. A 65, 033609 (2002).

[13] H.D. Politzer, Phys. Rev. A 54, 5048 (1996); P. Navez et al., Phys. Rev. Lett. 79, 1789 (1997); S. Grossmann and M. Holthaus, ibid, 3557 (1997).

[14] M.R. Andrews et al., Science 275, 637-641 (1997).

[15] C. Orzel et al., Science 291, 2386 (2001).

[16] M. Greiner et al., Nature 413, 44 (2002). M. Greiner et al., Nature 419, 51 (2002).

[17] D. Jaksch et al., Phys. Rev. Lett. 81, 3108 (1998).

[18] D. M. Stemper-Kurn et al., Phys. Rev. Lett. 80, 2027 (1998).

[19] M. Barrett, J. Sauer, and M. S. Chapman, Phys. Rev.
Lett. 87, 010404 (2001).

[20] J. A. Dunningham and K. Burnett, Phys. Rev. Lett. 82, 3729 (1999).

[21] J. Javanainen, J. Phys. B 33, 5493 (2000); S. Ashhab and A. J. Leggett, Phys. Rev. A 65, 023604 (2002).

[22] S. Yi et al., Phys. Rev. A 66, 011601(R) (2002).

[23] T.-L. Ho, Phys. Rev. Lett. 81, 742 (1998).

[24] T. Ohmi and K. Machida, J. Phys. Soc. Jap. 67, 1822 (1998).

[25] H. Pu, C. K. Law, and N. P. Bigelow, Physica B 280, 27, (2000); C. K. Law, H. Pu, and N. P. Bigelow, Phys. Rev. Lett. 81, 5257 (1998).

[26] E. Goldstein and P. Meystre, Phys. Rev. A 59, 3896 (1999).

[27] P. Nozieres and D. Pines, The Theory of Quantum Liquids, (Addison-Wesley, Reading, MA, 1990), Vol. 2.

[28] S. Yi, Ö. E. Müstecaplığlu, and L. You, (unpublished).

[29] T. Isoshima et al., Phys. Rev. A 60, 4857 (1999); N.P. Robins et al., Phys. Rev. A 64, 021601(R) (2001).

[30] M. Ueda, Phys. Rev. A 63, 013601 (2000).

[31] Ö. E. Müstecaplıŏlu, M. Zhang, and L. You, Phys. Rev. A 66, 033611 (2002).

[32] F. Dalfovo, L. Pitaevskii, and S. Stringari, Phys. Rev. A 54, 4213 (1996). 


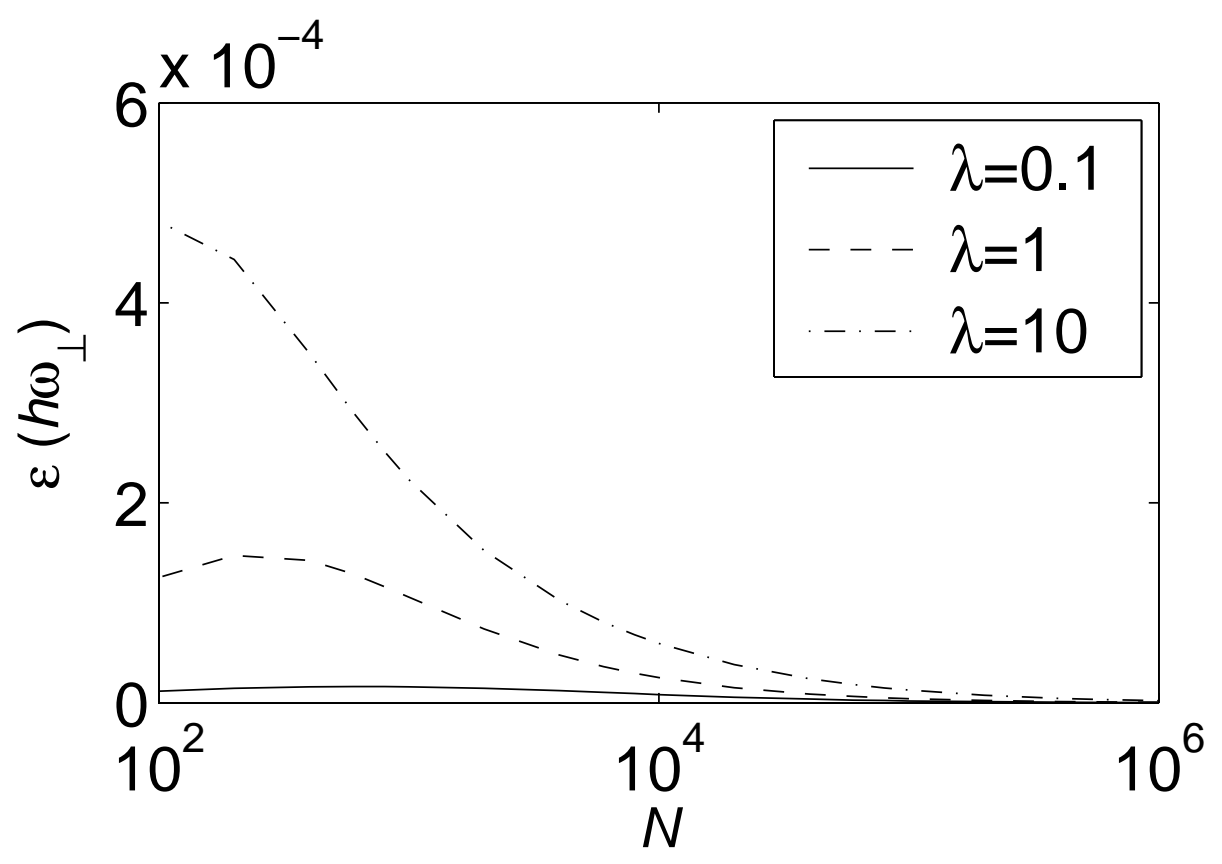

